\title{
Association between enterocyte injury and fluid balance in patients with septic shock: a post hoc exploratory analysis of a prospective observational study
}

Haruka Yokoyama ${ }^{1}$, Motohiro Sekino ${ }^{1 *}$, Hiroyuki Funaoka ${ }^{2}$, Shuntaro Sato ${ }^{3}$, Hiroshi Araki ${ }^{1}$, Takashi Egashira ${ }^{4}$, Rintaro Yano', Sojiro Matsumoto', Taiga Ichinomiya', Ushio Higashijima and Tetsuya Hara'

\begin{abstract}
Background: The required fluid volume differs among patients with septic shock. Enterocyte injury caused by shock may increase the need for fluid by triggering a systematic inflammatory response or an ischemia-reperfusion injury in the presence of intestinal ischemia/necrosis. This study aimed to evaluate the association between enterocyte injury and positive fluid balance in patients with septic shock.

Methods: This study was a post hoc exploratory analysis of a prospective observational study that assessed the association between serum intestinal fatty acid-binding protein, a biomarker of enterocyte injury, and mortality in patients with septic shock. Intestinal fatty acid-binding protein levels were recorded on intensive care unit admission, and fluid balance was monitored from intensive care unit admission to Day 7. The association between intestinal fatty acid-binding protein levels at admission and the infusion balance during the early period after intensive care unit admission was evaluated. Multiple linear regression analysis, with adjustments for severity score and renal function, was performed.
\end{abstract}

Results: Overall, data of 57 patients were analyzed. Logarithmically transformed intestinal fatty acid-binding protein levels were significantly associated with cumulative fluid balance per body weight at 24 and $72 \mathrm{~h}$ post-intensive care unit admission both before (Pearson's $r=0.490$ [95\% confidence interval: 0.263-0.666]; $P<0.001$ and $r=0.479$ [95\% confidence interval: 0.240-0.664]; $P<0.001$, respectively) and after (estimate, 14.4 [95\% confidence interval: 4.1-24.7]; $P=0.007$ and estimate, 26.9 [95\% confidence interval: $11.0-42.7$ ]; $P=0.001$, respectively) adjusting for severity score and renal function.

Conclusions: Enterocyte injury was significantly associated with cumulative fluid balance at 24 and $72 \mathrm{~h}$ post-intensive care unit admission. Enterocyte injury in patients with septic shock may be related to excessive fluid accumulation during the early period after intensive care unit admission.

Keywords: Enterocyte damage, Fluid administration, Fluid volume, Intensive care unit, Intestinal fatty acid-binding protein, Intestinal mucosal cell, Sepsis, Septic shock

\footnotetext{
*Correspondence: m-sekino@nagasaki-u.ac.jp

1 Department of Anesthesiology and Intensive Care Medicine, Nagasaki

University Graduate School of Biomedical Sciences, 1-7-1 Sakamoto, Nagasaki 852-8501, Japan

Full list of author information is available at the end of the article
}

\section{Background}

Sepsis is responsible for considerable morbidity and mortality, with most deaths occurring due to cardiovascular or multiorgan failure [1]. Fluid therapy is essential original author(s) and the source, provide a link to the Creative Commons licence, and indicate if changes were made. The images or other third party material in this article are included in the article's Creative Commons licence, unless indicated otherwise in a credit line to the material. If material is not included in the article's Creative Commons licence and your intended use is not permitted by statutory regulation or exceeds the permitted use, you will need to obtain permission directly from the copyright holder. To view a copy of this licence, visit http://creativecommons.org/licenses/by/4.0/. The Creative Commons Public Domain Dedication waiver (http://creativeco mmons.org/publicdomain/zero/1.0/) applies to the data made available in this article, unless otherwise stated in a credit line to the data. 
for early resuscitation in patients with septic shock to increase cardiac output and improve organ perfusion; however, optimal targets and fluid therapy strategies are yet to be established [2, 3].

Several studies have demonstrated an association between positive fluid balance and poor outcomes in patients with septic shock [4-7]. Recently, large database studies have also reported an association between prognosis and the amount of fluid infused or fluid balance in the early period of intensive care unit (ICU) admission, especially within $24-72 \mathrm{~h}[8,9]$. Fluid overload causes multiple organ dysfunction due to edema in the pulmonary, renal, and cardiovascular systems, worsening the prognosis [10]. Therefore, it stands to reason that fluid volume restriction may improve prognosis. However, the effects of such restrictions remain unproven [11]. Infusion of excess fluid that deviates from the appropriate preload should be avoided, and fluid restriction must be applied to improve the prognosis. However, other factors such as enterocyte injury may promote a positive fluid balance due to increased fluid requirements and may also be associated with a poor prognosis.

Enterocytes comprise more than $80 \%$ of all intestinal epithelial cells and act as a barrier to translocation of luminal antigens, and microbiota and their toxic products into the circulation [12]. Hypoperfusion injury of the enterocytes destroys this barrier and triggers a systemic inflammatory response, which can lead to multiple organ dysfunction and poor outcomes [13-15]. Enterocyte injury itself may increase the need for fluid by triggering a systemic inflammatory response or ischemiareperfusion injury when resulting intestinal ischemia or necrosis occurs [16]. In other words, patients with septic shock who present with enterocyte injury may have a more positive fluid balance than those without enterocyte injury. However, to our knowledge, the association between enterocyte injury and positive fluid balance in patients with septic shock has not been evaluated. If an association is identified, it may help individualize strategies to promote an optimal fluid balance in patients with septic shock.

We investigated the relationship between enterocyte injury in patients with septic shock and fluid balance during the early period after ICU admission. We hypothesized that, in patients with septic shock, enterocyte injury at ICU admission is associated with an excessive positive fluid balance $24-$ and 72 -h post-ICU admission.

\section{Methods}

\section{Study design}

This was a post hoc exploratory analysis of data obtained from a prospective observational study that evaluated the association between enterocyte injury and patient outcomes in patients with septic shock [17, 18]. Additional fluid management data were collected, along with the original study's data, and additional statistical tests were performed to address new clinical questions. The original study was conducted in an eight-bed general ICU at Nagasaki University Hospital, Nagasaki, Japan, between May 2012 and March 2015. Ethical approval for this post hoc analysis was obtained from the Institutional Review Board of Nagasaki University Hospital (approval number: 18111922). The requirement for written informed consent was waived owing to the retrospective nature of the study.

\section{Study population}

A total of 57 patients with septic shock, who required mechanical ventilation, were enrolled in the original study. Septic shock was defined as acute organ dysfunction in the presence of infection with a need for vasopressors [19]. The original study excluded patients with confirmed or strongly suspected small bowel ischemia with or without necrosis at the source of sepsis, a medical history of extensive small bowel resection, chronic small bowel disease, pregnancy, uncontrolled bleeding, and those in the terminal stages of any comorbidity.

\section{Data collection}

The database from the original study and newly collected data were used in this study. The baseline values at ICU admission and the values within the first $24 \mathrm{~h}$ of admission were taken from the original study's database, which included age, sex, body mass index, Acute Physiology and Chronic Health Evaluation (APACHE) II score, Sequential Organ Failure Assessment score, Japanese Association for Acute Care Medicine (JAAM) disseminated intravascular coagulation (DIC) score [20], mean arterial pressure, heart rate (HR), central venous pressure (CVP), inotropic score [21], blood gas analysis data $\left(\mathrm{PaO}_{2} / \mathrm{F}_{\mathrm{I}} \mathrm{O}_{2}\right.$ ratio, $\mathrm{pH}$, and $\mathrm{HCO}_{3}{ }^{-}$), ventilator settings (positive endexpiratory pressure and peak inspiratory pressure), and post-admission interventions for septic shock management, including the administration of vasopressin, lowdose steroids, continuous renal replacement therapy, or polymyxin B-direct hemoperfusion. Estimated glomerular filtration rate (eGFR) at ICU admission was calculated using the equation for Japanese patients recommended by the Japanese Society of Nephrology: eGFR $\left(\mathrm{mL} / \mathrm{min} / 1.73 \mathrm{~m}^{2}\right)=194 \times \mathrm{Cr}(\mathrm{mg} / \mathrm{dL})^{-1.094} \times$ age $^{-0.287} \quad$ (if female, $\times 0.739$ ) [22]. Admission route, surgical interventions, site of infection, and bacteremia status were also included. Patient outcomes, including all-cause 28-day mortality and in-hospital mortality, were recorded.

Standard blood chemistry tests were performed at ICU admission to evaluate sepsis severity, including 
arterial levels of serum lactate, N-terminal pro-B-type natriuretic peptide, and procalcitonin. These data were obtained from records maintained by Nagasaki University Hospital.

We collected retrospective data on fluid management from the ICU information system (Prescient ICU; FUJIFILM Medical Co. Ltd., Tokyo, Japan). Data were collected for the first 7 days of ICU admission. The first day of ICU admission was defined as the time between ICU admission and $24 \mathrm{~h}$. Daily fluid intake was calculated as the sum of all intravenous and oral fluids (including blood products and nutritional supplements). Daily fluid output was calculated as the sum of the urine volume, ultrafiltration volume, drainage volume, and gastrointestinal loss. Daily fluid balance was calculated by subtracting the total fluid output from the total fluid intake. For patients who died or were discharged from the ICU within 7 days, the fluid balance was calculated up to the day before the date of death or discharge.

\section{Assessment of enterocyte injury}

Serum intestinal fatty acid-binding protein (I-FABP) was used as a biomarker of enterocyte injury. Serum I-FABP levels were provided by an external laboratory (DS Pharma Biomedical [now SB Bioscience] Co. Ltd., Osaka, Japan) using a human I-FABP-specific enzyme-linked immunosorbent assay. The mean serum I-FABP level in healthy adult volunteers was $1.1 \pm 0.9 \mathrm{ng} / \mathrm{mL}$ (range, $0.1-5.5$ ) [23], and the cutoff value for diagnosing small bowel ischemia was $9.1 \mathrm{ng} / \mathrm{mL}$ [24]. ICU physicians were blinded to the patients' I-FABP levels.

\section{Clinical management}

During the ICU stay, the fluid management plan was determined at the discretion of experienced ICU physicians. Fluid management was based on echocardiography, volume responsiveness to blood pressure and HR, and CVP, as appropriate; however, there was no specific, standardized fluid management plan. Blood products were used based on experienced ICU physicians' orders.

Details regarding the patients' clinical management have been described previously [17, 18]. All patients were principally treated as per the Surviving Sepsis Campaign guidelines [19], except for Japan-specific treatment options, such as polymyxin B-direct hemoperfusion [25], and the administration of recombinant soluble thrombomodulin and antithrombin for septic DIC [26, 27].

\section{Statistical analysis}

Clinical data are presented as medians and interquartile ranges for quantitative variables and as frequencies and percentages for categorical variables. I-FABP levels and eGFR were logarithmically transformed to normalize their distributions. The correlation between cumulative fluid balance per body weight at 24- and 72-h post-ICU admission and I-FABP levels at ICU admission were evaluated using Pearson's correlation coefficient (r). A multiple linear regression analysis was performed to evaluate the association between cumulative fluid balance per body weight at 24- and 72-h post-ICU admission and I-FABP levels at ICU admission. The non-renal APACHE II score (APACHE II score without the renal component) and eGFR at ICU admission were adopted as confounders. The APACHE II score was used as the sepsis severity score and was directly suggestive of fluid balance [9]. Furthermore, eGFR was used to assess renal function, as it is associated with I-FABP clearance and affects I-FABP levels [28]. All tests were two-sided, and $P$-values $<0.05$ were considered statistically significant. All statistical analyses were performed using JMP Pro v15 (SAS Institute Inc., Cary, NC, USA) and R version 3.5.0 (R Foundation for Statistical Computing, Vienna, Austria).

\section{Results \\ Study population}

Fifty-seven patients with septic shock, who required mechanical ventilation, were included in this post hoc analysis. Their baseline characteristics are summarized in Table 1. Most patients were older and had a more severe shock. The proportion of patients diagnosed with DIC using the JAAM DIC score was $72 \%$. Eleven patients had hospital-onset septic shock, while the remaining 46 patients had an out-of-hospital shock. Twenty-two patients required surgery for source control before ICU admission. The most common source of infection was the abdomen (33\%), followed by the lung/thorax (21\%), soft tissues (16\%), urinary tract (7\%), and others (23\%). Blood cultures were positive in $54 \%$ of the patients. The overall 28 -day and in-hospital mortality rates were $23 \%(13 / 57)$ and $32 \%(18 / 57)$, respectively.

\section{Daily fluid balance and I-FABP levels over the first week of ICU admission}

Figure 1 illustrates the median daily fluid balance and I-FABP levels over the first week of ICU admission. Positive fluid balances were observed during the early post-ICU admission period, especially on ICU Day 1. However, an almost even balance was observed after ICU Day 3. The median cumulative fluid balance values on ICU Day 1 ( $24 \mathrm{~h}$ after ICU admission) and ICU Day 3 (72 $\mathrm{h}$ after ICU admission) were 4390 (2588-6636) $\mathrm{mL}$ and $6409(4018-11,531) \mathrm{mL}$, respectively. I-FABP levels rapidly decreased after ICU admission. 
Table 1 Baseline characteristics of patients with septic shock

\begin{tabular}{|c|c|}
\hline Characteristics & Median (IQR) \\
\hline Age, years & $71(62-79)$ \\
\hline Male, n (\%) & $35(61)$ \\
\hline Body mass index, $\mathrm{kg} / \mathrm{m}^{2}$ & $22(19-24)$ \\
\hline APACHE II score & $30(25-36)$ \\
\hline SOFA score & $13(11-15)$ \\
\hline JAAM DIC score & $5(3-7)$ \\
\hline \multicolumn{2}{|l|}{ Baseline data and setting at ICU admission } \\
\hline Mean arterial pressure, $\mathrm{mmHg}$ & $81(73-90)$ \\
\hline Heart rate, beats/min & $103(92-113)$ \\
\hline Central venous pressure, $\mathrm{mmHg}$ & $11(9-14)$ \\
\hline Inotropic score ${ }^{a}$ & $40(25-55)$ \\
\hline $\mathrm{PaO}_{2} / \mathrm{F}_{1} \mathrm{O}_{2}$ ratio & $227(174-290)$ \\
\hline PEEP, $\mathrm{cmH}_{2} \mathrm{O}$ & $8(5-8)$ \\
\hline Peak inspiratory pressure, $\mathrm{cmH}_{2} \mathrm{O}$ & $21(20-23)$ \\
\hline $\mathrm{pH}$ & $7.29(7.26-7.34)$ \\
\hline $\mathrm{HCO}_{3}{ }^{-}, \mathrm{mmol} / \mathrm{L}$ & $17.7(15.5-20.2)$ \\
\hline Lactate, $\mathrm{mmol} / \mathrm{L}$ & $3.0(2.1-6.8)$ \\
\hline Procalcitonin, ng/mL & $42.5(10.7-118.2)$ \\
\hline NT-proBNP, ng/mL & $9585(2388-24,389)$ \\
\hline $\mathrm{eGFR}, \mathrm{mL} / \mathrm{min} / 1.73 \mathrm{~m}^{2 \mathrm{~b}}$ & $20(11-38)$ \\
\hline \multicolumn{2}{|l|}{ Intervention after ICU admission } \\
\hline Vasopressin, n (\%) & $28(49)$ \\
\hline Steroid, n (\%) & $46(81)$ \\
\hline Continuous renal replacement therapy, n (\%) & $46(81)$ \\
\hline Polymyxin B-direct hemoperfusion, $\mathrm{n}(\%)$ & $31(54)$ \\
\hline
\end{tabular}

Abbreviations: APACHE Acute Physiology and Chronic Health Evaluation, DIC Disseminated intravascular coagulation, eGFR Estimated glomerular filtration rate, ICU Intensive care unit, IQR Interquartile range, JAAM Japanese Association for Acute Care Medicine, NT-proBNP N-terminal pro-B-type natriuretic peptide, PEEP Positive end-expiratory pressure, SOFA Sequential Organ Failure Assessment

Data are reported as median (IQR), unless otherwise indicated

${ }^{a}$ Inotropic score calculated as (dopamine dose $\times 1$ ) + (dobutamine dose $\times 1)+($ epinephrine dose $\times 100)+($ norepinephrine dose $\times 100)$, where all doses are expressed in micrograms per kilogram per minute

b eGFR calculated as $194 \times \mathrm{Cr}(\mathrm{mg} / \mathrm{dL})^{-1.094} \times$ age $^{-0.287}$ (if female, $\left.\times 0.739\right)$

\section{Correlation between I-FABP levels at ICU admission and cumulative fluid balance at 24 and 72 hours after ICU admission}

At ICU admission, I-FABP levels were significantly correlated with the 24- and 72-h cumulative fluid balance (Pearson's $r=0.490$ [95\% confidence interval (CI): $0.263-0.666$ ] $P<0.001$ and $r=0.479$ [95\% CI: $0.240-$ 0.664 ]; $P<0.001$, respectively) (Fig. $2 \mathrm{a}, \mathrm{b})$.
Association between fluid balance at 24 and 72 hours and I-FABP levels at ICU admission adjusted for severity score and renal function

The results of multiple linear regression analysis of cumulative fluid balance per body weight at 24- and 72-h post-ICU admission and I-FABP levels at ICU admission, adjusted for non-renal APACHE II score and eGFR, are summarized in Table 2. I-FABP levels were significantly associated with cumulative fluid balance at both 24 (estimate, 14.4 [95\% CI: 4.1-24.7]; $P=0.007$ ) and $72 \mathrm{~h}$ (estimate, 26.9 [95\% CI: 11.0-42.7]; $P=0.001)$ post-ICU admission. In other words, a $1 \%$ increase in the I-FABP level increases the cumulative infusion balance by $0.144 \mathrm{~mL}$ per $\mathrm{kg}$ of body weight at $24 \mathrm{~h}$ and by $0.269 \mathrm{~mL}$ per $\mathrm{kg}$ of body weight at $72 \mathrm{~h}$ after ICU admission.

\section{Discussion}

I-FABP level at ICU admission was significantly associated with cumulative fluid balance at 24 - and 72 -h postICU admission, both before and after adjusting for sepsis severity score and renal function. In patients experiencing septic shock, enterocyte injury may be related to an excessive positive fluid balance during the early period after ICU admission.

Fluid requirements vary widely among patients, and factors associated with increased fluid requirements in patients with septic shock remain unclear. In a recent study, which reported an association between the volume of fluid infused during the first $24 \mathrm{~h}$ after ICU admission and prognosis, a large range of fluid volumes were administered during the first $24 \mathrm{~h}$ after ICU admission; these volumes ranged from $2000 \mathrm{~mL}$ (10th percentile) to $9288 \mathrm{~mL}$ (90th percentile) among patients with septic shock who required mechanical ventilation [8]. In addition to more severe illness, older age, postoperative and immunosuppressed status, positive blood cultures, and sepsis with an abdominal source of infection were cited as factors potentially related to a positive fluid balance in the first $24 \mathrm{~h}$ after ICU admission [9]. Since intestinal ischemia/necrosis is assumed to be the commonest source of abdominal infection, we believe that this result supports our findings. Enterocyte injury followed by a systemic inflammatory response and possible intestinal ischemia/necrosis may significantly affect the fluid balance during the early period after ICU admission.

To improve the prognosis of patients with septic shock, iatrogenic fluid overload should be avoided. However, uniform fluid restrictions in patients with increased fluid requirements may lead to hypovolemia and a worse prognosis. Studies that evaluated the effectiveness of protocol-based fluid restriction reported that interventions 

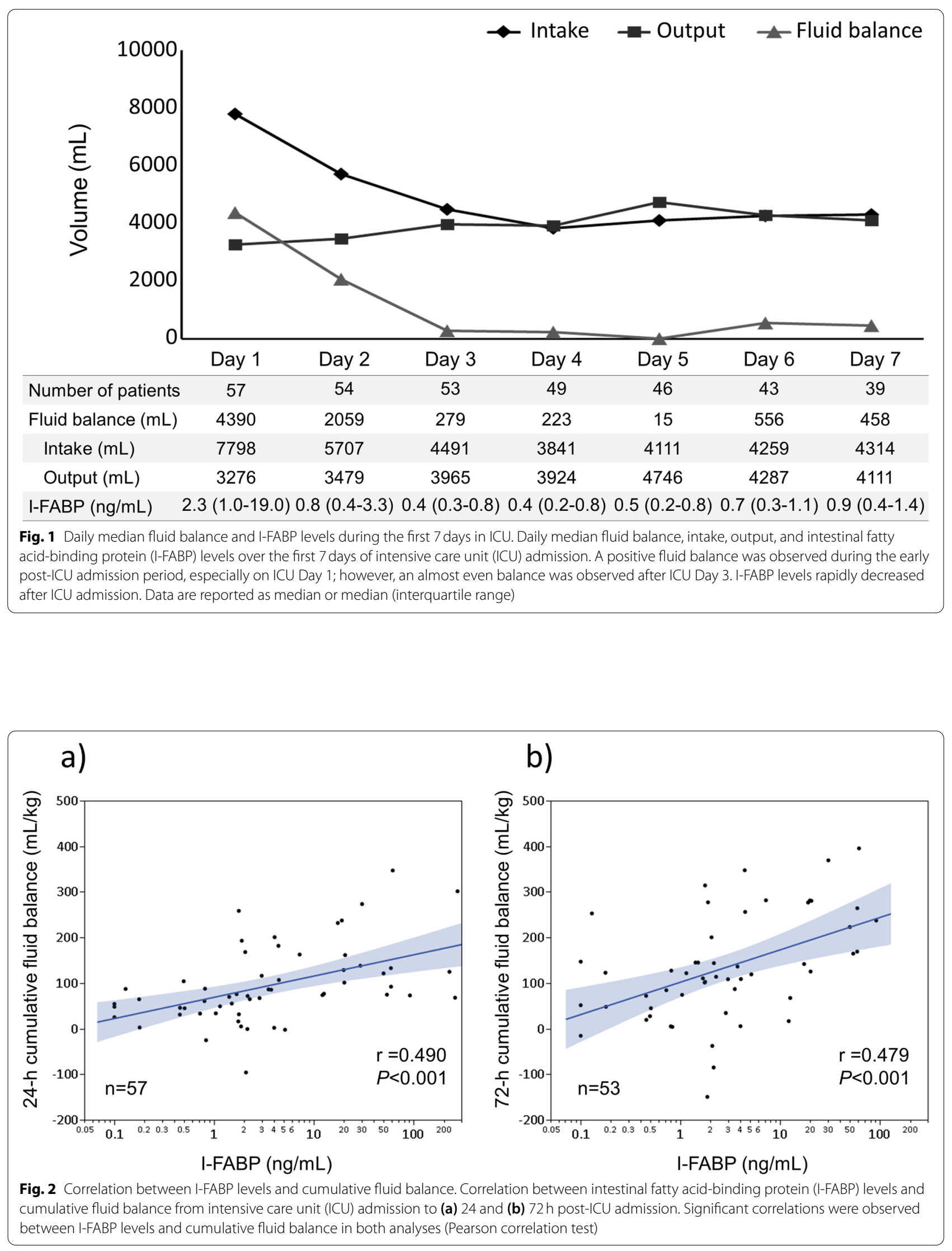
Table 2 Multiple linear regression analysis of fluid balance and I-FABP adjusted for severity score and eGFR

\begin{tabular}{|c|c|c|c|c|}
\hline \multirow{3}{*}{$\begin{array}{l}\text { Dependent } \\
\text { variables }\end{array}$} & \multicolumn{4}{|c|}{ Independent variables: log I-FABP } \\
\hline & \multirow[t]{2}{*}{ Estimate $^{a}$} & \multicolumn{2}{|l|}{$95 \% \mathrm{Cl}$} & \multirow[t]{2}{*}{$P$-value } \\
\hline & & Lower limit & Upper limit & \\
\hline $\begin{array}{l}\text { 24-h fluid balance/ } \\
\text { BW }\end{array}$ & 14.4 & 4.1 & 24.7 & $0.007^{b}$ \\
\hline $\begin{array}{l}\text { 72-h fluid balance/ } \\
\text { BW }\end{array}$ & 26.9 & 11.0 & 42.7 & $0.001^{b}$ \\
\hline
\end{tabular}

Abbreviations: APACHE Acute Physiology and Chronic Health Evaluation, BW Body weight, $\mathrm{Cl}$ Confidence interval, eGFR Estimated glomerular filtration rate, I-FABP Intestinal fatty acid-binding protein

a Adjusted for non-renal APACHE II score and log eGFR

b Statistically significant

could suppress infusion balance and reduce the incidence of renal injury $[29,30]$; however, the effects of such a protocol on prognosis are unknown [11]. Additionally, protocol violations and deviations were reported in the infusion-restricted group [29, 30]. These violations could have resulted from the physicians' opinions that infusion restrictions did not benefit some of their patients. There is an urgent need to identify factors associated with increased fluid requirements in patients with septic shock to provide individualized fluid management strategies.

Enterocyte injury is reportedly associated with poor clinical outcomes in patients with critical illness [31], acute heart failure, cardiogenic shock [32], postoperative cardiac surgery [33, 34], and septic shock-as demonstrated in our original study $[17,18]$. Enterocyte injury increases the fluid requirements and may be associated with a worse prognosis. Our original study revealed that I-FABP levels at ICU admission were associated with 28-day mortality and the incidence of non-occlusive mesenteric ischemia [17]. In patients who present with enterocyte injury, gut-derived sepsis and hidden intestinal ischemia/necrosis should be addressed while optimizing infusion volume to improve prognosis. However, the question of whether enterocyte injury is an independent risk factor associated with increased fluid requirements and poor prognosis should be elucidated in future, well-powered studies.

To our knowledge, this is the first report investigating the association between enterocyte injury at ICU admission and fluid balance during the early period after ICU admission. An excessive positive fluid balance can be caused by patient-related factors, as well as iatrogenic fluid overload. Elucidation of patient-related factors is necessary to determine individual fluid needs and improve the prognosis of patients with septic shock. We believe that this study is crucial because our findings suggest that enterocyte injury may be one such factor with prognostic significance.

This study has several limitations. First, there are inherent limitations to post hoc exploratory analyses, and our patient cohort was relatively small. Therefore, our findings require confirmation through the prospective examination of a larger cohort. Second, we did not have access to information about the patients' pre-ICU admission fluid volumes and outputs. It was difficult to retrospectively collect accurate data before admission to our hospital, either in the ward or emergency room. At ICU admission, our patients' mean arterial pressure, HR, and CVP values were within a relatively narrow range; therefore, we presume that there were no significant differences in their respective pre-admission fluid needs. Although differences in fluid balance before ICU admission may have affected our results, other studies have likewise analyzed fluid balance solely after ICU admission [4-9]. Third, the median CVP value on ICU admission in this study was relatively high; therefore, congestion may have caused the enterocyte injury. A study examining the association between venous congestion, assessed by invasively measured hemodynamic parameters, and enterocyte injury in patients with acute decompensated heart failure found no association between them [35]. If enterocyte injury was caused by congestion due to excessive fluid infusion before and after ICU admission, the I-FABP levels should have increased at ICU admission or after ICU admission; however, as shown in Fig. 1, the I-FABP levels decreased after ICU admission, and there was no correlation between CVP at ICU admission and I-FABP levels at ICU admission in this study (Pearson's $r=-0.035 ; P=0.797)$. Theoretically, congestion can also cause small enterocyte injury; however, we believe that the primary cause of enterocyte injury in this study was hypoperfusion due to septic shock.

\section{Conclusions}

Our post hoc exploratory analysis found that enterocyte injury at ICU admission was significantly correlated with fluid balance, particularly during the early periodwithin 24 and $72 \mathrm{~h}$-post-ICU admission, both before and after adjusting for sepsis severity and renal function. In patients with septic shock, enterocyte injury may contribute to excessive fluid accumulation during the early period after ICU admission.

\section{Abbreviations}

APACHE: Acute Physiology and Chronic Health Evaluation; Cl: Confidence interval; CVP: Central venous pressure; DIC: Disseminated intravascular coagulation; eGFR: Estimated glomerular filtration rate; HR: Heart rate; ICU: Intensive care unit; I-FABP: Intestinal fatty acid-binding protein; JAAM: Japanese Association for Acute Care Medicine. 


\section{Acknowledgments}

We would like to thank Editage (www.editage.jp) for their writing support.

\section{Authors' contributions}

$\mathrm{HY}, \mathrm{MS}, \mathrm{UH}, \mathrm{TI}$, and TH contributed to the study concept and design. HY, MS, $H F, U H, T I, S M, R Y, T E$, and $H A$ contributed to the acquisition, analysis, and interpretation of data. SS performed the statistical analyses. HY drafted the manuscript. MS and TH critically revised the manuscript for important intellectual content. All authors read and approved the final manuscript.

\section{Funding}

This work was supported in part by the Japan Society for the Promotion of Science (KAKENHI; grant numbers: 23791713, 16 K10942, and 20 K09220, awarded to MS).

\section{Availability of data and materials}

The datasets used and analyzed during the current study are available from the corresponding author on reasonable request.

\section{Declarations}

\section{Ethics approval and consent to participate}

All procedures performed in studies involving human participants were in accordance with the ethical standards of the institutional and national research committee and with the 1964 Declaration of Helsinki and its later amendments or comparable ethical standards. The study design was approved by the Institutional Review Board of Nagasaki University Hospital (approval number: 18111922). The requirement for written informed consent was waived owing to the retrospective nature of the study.

\section{Consent for publication}

Not applicable.

\section{Competing interests}

The authors declare that they have no competing interests.

\section{Author details}

${ }^{1}$ Department of Anesthesiology and Intensive Care Medicine, Nagasaki University Graduate School of Biomedical Sciences, 1-7-1 Sakamoto, Nagasaki 852-8501, Japan. ${ }^{2}$ Department of Research and Development, SB Bioscience Co. Ltd., 3-47 Higashi-Tsukaguchi-cho, 2-chome, Amagasaki, Hyogo 661-0011, Japan. ${ }^{3}$ Clinical Research Center, Nagasaki University Hospital, 1-7-1 Sakamoto, Nagasaki 852-8501, Japan. ${ }^{4}$ Department of Intensive Care, Nagasaki Harbor Medical Center, 6-39 Shinchi-machi, Nagasaki 850-8555, Japan.

\section{Received: 17 June 2021 Accepted: 15 November 2021}

Published online: 23 November 2021

\section{References}

1. Cecconi M, Evans L, Levy M, Rhodes A. Sepsis and septic shock. Lancet. 2018;392:75-87.

2. Evans L, Rhodes A, Alhazzani W, Antonelli M, Coopersmith CM, French $C$, et al. Surviving sepsis campaign: international guidelines for management of sepsis and septic shock 2021. Intensive Care Med. 2021:47:1181-247.

3. Egi M, Ogura H, Yatabe T, Atagi K, Inoue S, Iba T, et al. The Japanese clinical practice guidelines for management of sepsis and septic shock 2020 (J-SSCG 2020). J Intensive Care. 2021;9:53.

4. Boyd JH, Forbes J, Nakada TA, Walley KR, Russell JA. Fluid resuscitation in septic shock: a positive fluid balance and elevated central venous pressure are associated with increased mortality. Crit Care Med. 2011;39:259-65

5. Acheampong A, Vincent JL. A positive fluid balance is an independent prognostic factor in patients with sepsis. Crit Care. 2015;19:251.

6. de Oliveira FS, Freitas FG, Ferreira EM, de Castro I, Bafi AT, de Azevedo LC, et al. Positive fluid balance as a prognostic factor for mortality and acute kidney injury in severe sepsis and septic shock. J Crit Care. 2015:30:97-101.
7. Wang YM, Chen Y, Zheng YJ, Huang YC, Chen WW, Ji R, et al. Low fluid intake volume during the first $24 \mathrm{~h}$ and persistent negative fluid balance from the second day are associated with favorable prognosis for patients with sepsis. Exp Ther Med. 2021;21:387.

8. Marik PE, Linde-Zwirble WT, Bittner EA, Sahatjian J, Hansell D. Fluid administration in severe sepsis and septic shock, patterns and outcomes: an analysis of a large national database. Intensive Care Med. 2017:43:625-32.

9. Sakr Y, Rubatto Birri PN, Kotfis K, Nanchal R, Shah B, Kluge S, et al. Higher fluid balance increases the risk of death from sepsis: results from a large international audit. Crit Care Med. 2017;45:386-94.

10. Jaffee W, Hodgins S, McGee WT. Tissue edema, fluid balance, and patient outcomes in severe sepsis: an organ systems review. J Intensive Care Med. 2018:33:502-9.

11. Meyhoff TS, Møller MH, Hjortrup PB, Cronhjort M, Perner A, Wetterslev J. Lower vs higher fluid volumes during initial management of sepsis: a systematic review with meta-analysis and trial sequential analysis. Chest. 2020;157:1478-96.

12. Derikx JP, Luyer MD, Heineman E, Buurman WA. Non-invasive markers of gut wall integrity in health and disease. World J Gastroenterol. 2010;16:5272-9.

13. Clark JA, Coopersmith CM. Intestinal crosstalk: a new paradigm for understanding the gut as the "motor" of critical illness. Shock. 2007;28:384-93.

14. Mittal R, Coopersmith CM. Redefining the gut as the motor of critical illness. Trends Mol Med. 2014;20:214-23.

15. Assimakopoulos SF, Triantos C, Thomopoulos K, Fligou F, Maroulis I, Marangos M, et al. Gut-origin sepsis in the critically ill patient: pathophysiology and treatment. Infection. 2018;46:751-60.

16. Doig CJ, Sutherland LR, Sandham JD, Fick GH, Verhoef M, Meddings JB. Increased intestinal permeability is associated with the development of multiple organ dysfunction syndrome in critically ill ICU patients. Am J Respir Crit Care Med. 1998;158:444-51.

17. Sekino M, Funaoka H, Sato S, Okada K, Inoue H, Yano R, et al. Intestinal fatty acid-binding protein level as a predictor of 28-day mortality and bowel ischemia in patients with septic shock: a preliminary study. J Crit Care. 2017:42:92-100.

18. Sekino M, Funaoka H, Sato S, Okada K, Inoue H, Yano R, et al. Association between macroscopic tongue ischemia and enterocyte injury and poor outcome in patients with septic shock: a preliminary observational study. Shock. 2018;50:530-7

19. Dellinger RP, Levy MM, Rhodes A, Annane D, Gerlach H, Opal SM, et al. Surviving sepsis campaign: international guidelines for management of severe sepsis and septic shock: 2012. Crit Care Med. 2013;41:580-637.

20. Gando S, Saitoh D, Ogura H, Fujishima S, Mayumi T, Araki T, et al. A multicenter, prospective validation study of the Japanese Association for Acute Medicine disseminated intravascular coagulation scoring system in patients with severe sepsis. Crit Care. 2013;17:R111.

21. Cruz DN, Antonelli M, Fumagalli R, Foltran F, Brienza N, Donati A, et al. Early use of polymyxin $B$ hemoperfusion in abdominal septic shock: the EUPHAS randomized controlled trial. JAMA. 2009;301:2445-52.

22. Matsuo S, Imai E, Horio M, Yasuda Y, Tomita K, Nitta K, et al. Revised equations for estimated GFR from serum creatinine in Japan. Am J Kidney Dis. 2009;53:982-92.

23. Funaoka H, Kanda T, Kajiura S, Ohkaru Y, Fujii H. Development of a high-specificity sandwich ELISA system for the quantification of human intestinal fatty acid-binding protein (I-FABP) concentrations. Immunol Investig. 2011;40:223-42.

24. Matsumoto S, Sekine K, Funaoka H, Yamazaki M, Shimizu M, Hayashida $K$, et al. Diagnostic performance of plasma biomarkers in patients with acute intestinal ischaemia. Br J Surg. 2014;101:232-8.

25. Nakamura Y, Kitamura T, Kiyomi F, Hayakawa M, Hoshino K, Kawano $Y$, et al. Potential survival benefit of polymyxin $B$ hemoperfusion in patients with septic shock: a propensity-matched cohort study. Crit Care. 2017;21:134.

26. Yamakawa K, Aihara M, Ogura H, Yuhara H, Hamasaki T, Shimazu T. Recombinant human soluble thrombomodulin in severe sepsis: a systematic review and meta-analysis. J Thromb Haemost. 2015;13:508-19.

27. Hayakawa M, Kudo D, Saito S, Uchino S, Yamakawa K, lizuka Y, et al. Antithrombin supplementation and mortality in sepsis-induced disseminated intravascular coagulation: a multicenter retrospective observational study. Shock. 2016;46:623-31. 
28. Okada K, Sekino M, Funaoka H, Sato S, Ichinomiya T, Murata H, et al. Intestinal fatty acid-binding protein levels in patients with chronic renal failure. J Surg Res. 2018;230:94-100.

29. Hjortrup PB, Haase N, Bundgaard H, Thomsen SL, Winding R, Pettilä V, et al. Restricting volumes of resuscitation fluid in adults with septic shock after initial management: the classic randomised, parallel-group, multicentre feasibility trial. Intensive Care Med. 2016;42:1695-705.

30. Douglas IS, Alapat PM, Corl KA, Exline MC, Forni LG, Holder AL, et al. Fluid response evaluation in sepsis hypotension and shock: a randomized clinical trial. Chest. 2020;158:1431-45.

31. Piton G, Belon F, Cypriani B, Regnard J, Puyraveau M, Manzon C, et al. Enterocyte damage in critically ill patients is associated with shock condition and 28-day mortality. Crit Care Med. 2013;41:2169-76.

32. Kastl SP, Krychtiuk KA, Lenz M, Distelmaier K, Goliasch G, Huber K, et al. Intestinal fatty acid binding protein is associated with mortality in patients with acute heart failure or cardiogenic shock. Shock. 2019;51:410-5.
33. Zou L, Song X, Hong L, Shen X, Sun J, Zhang C, et al. Intestinal fatty acid-binding protein as a predictor of prognosis in postoperative cardiac surgery patients. Medicine (Baltimore). 2018;97:e11782.

34. Sekino M, Okada K, Funaoka H, Sato S, Ichinomiya T, Higashijima U, et al. Association between enterocyte injury and mortality in patients on hemodialysis who underwent cardiac surgery: an exploratory study. $J$ Surg Res. 2020;255:420-7.

35. Kitai T, Kim YH, Kiefer K, Morales R, Borowski AG, Grodin JL, et al. Circulating intestinal fatty acid-binding protein (I-FABP) levels in acute decompensated heart failure. Clin Biochem. 2017;50:491-5.

\section{Publisher's Note}

Springer Nature remains neutral with regard to jurisdictional claims in published maps and institutional affiliations.
Ready to submit your research? Choose BMC and benefit from:

- fast, convenient online submission

- thorough peer review by experienced researchers in your field

- rapid publication on acceptance

- support for research data, including large and complex data types

- gold Open Access which fosters wider collaboration and increased citations

- maximum visibility for your research: over $100 \mathrm{M}$ website views per year

At BMC, research is always in progress.

Learn more biomedcentral.com/submissions 